\title{
Joint Random Access and Power Selection for Maximal Throughput in Wireless Networks
}

\author{
Yan Gao, Zheng Zeng \\ \{yangao3, zzeng2\}@uiuc.edu \\ Department of Computer Science, and CSL \\ University of Illinois at Urbana-Champaign
}

\author{
P. R. Kumar \\ prkumar@illinois.edu \\ Department of Electrical and Computer Engineering, and CSL \\ University of Illinois at Urbana-Champaign
}

\begin{abstract}
In wireless networks, how to select transmit power that maximizes throughput is a challenging problem. On one hand, transmissions at a high power level could increase interference to others; on the other hand, transmissions at a low power level are prone to being interfered by others. Prior works consider this problem as a search for a fixed optimal power setting that maximizes communication spatial reuse. In this paper, we pursue a novel approach that combines power selection with a random medium access mechanism. For each transmission, a node randomly selects a transmit power from all available power levels to access the medium. In this way, all combinations of network power settings could be selected with some probability. Using a recently developed Markov chain model, we derive a distributed scheme that determines the access probabilities of each power setting, according to the arrival rate of traffic and the service rate achieved by the scheme. We show that this scheme always converges to the optimal solution. Moreover, we also show that the random scheme can attain the maximal throughput region that can be obtained by any time-sharing between power settings, and which is consequently larger than the region any fixed power setting can achieve.
\end{abstract}

\section{INTRODUCTION}

Power selection in wireless networks that chooses the transmit power for each packet is of foremost importance to maximize network capacity [5]. Since transmit power determines interference among wireless links [1], in principle it ought to be cooperatively selected so as to increase the level of spatial reuse. However, the problem is complex because changing power not only affects a local link's capability to tolerate interference but also alters its interference with others.

This problem has been recognized in [1], and extensively studied in [12], [3]. If one characterizes interference relationships by a conflict graph [6], then these existing approaches essentially pursue a fixed power assignment such that its corresponding conflict graph contains the largest independent set. In general, however, such a conflict graph may not be unique. For instance, consider the scenario in Figure 1. If link 1 chooses power level $p_{A}$ to transmit, then link 1 and link 3 can transmit concurrently. If link 1 chooses power level $p_{B}$, then link 1 and link 2 can transmit concurrently. The example shows that although the two conflict graphs are different, they

This material is based upon work partially supported by NSF under Contract Nos. CNS-07-21992, CNS-0626584, and ECCS-0701604, AFOSR under Contract FA9550-09-0121, and USARO under Contract Nos. W911NF08-1-0238 and W-911-NF-0710287. both allow two concurrent transmissions at the same time. The immediate question is which one is better? The answer should depend on the traffic demand on each wireless link; e.g., if link 3 has more traffic demand than link 2, the network will perform better with the second conflict graph. Furthermore, we argue that the optimal strategy should combine the two conflict graphs and schedule them according to the traffic demand.

Scheduling wireless links to achieve the maximal throughput, even for a fixed power configuration, is a hard problem [6]. A few deterministic schemes have been proposed, e.g., [15], [10]. There are also several randomized algorithms, e.g., [14], [11], [13], [2]. All the above scheduling schemes either work only for special interference models or require fully synchronized slotted systems, and therefore none of them works for asynchronous distributed networks. On the other hand, however, CSMA (carrier sense multiple access) type random access protocols are widely used in practice (e.g., IEEE802.11) because they can be easily implemented in a distributed manner. Recently, [9] proposed a Markov chain model to analyze the idealized CSMA-based random access protocols. Based on the model, [7] later developed an algorithm to adaptively change the attempt probability to meet the traffic demand without explicitly knowing the arrival rates. However, all these scheduling algorithms assume a fixed power assignment, and hence a fixed conflict graph. As discussed above, incorporating power control into scheduling could further improve the throughput.

In this paper, we introduce a random power access scheme that combines power control and multiple access control. The basic design of the scheme is similar to CSMA-based random access protocols. Let us assume that transmit power is chosen from a finite number of power levels. Before transmitting a packet, a node (sender of a link) senses whether the channel is busy. When the node detects that the channel is idle, it randomly initializes a back-off timer for each power level, and when the back-off timer reaches zero, transmits at the corresponding power level. While the node is transmitting, other power levels suspend their back-off processes and resume them when the channel is idle again. Based on the same model as in [9], [7], we derive a fully distributed algorithm that adaptively changes the parameters of the random access mechanism according to the local traffic demand. We show that the scheme can achieve the maximal throughput region 
in the sense that the network is stabilized for any arrival rate that can be served by some time-sharing between power settings, and which is consequently larger than that any fixed power setting can achieve. Therefore, the random power access scheme can serve the traffic rates that are not feasible with any of the fixed power assignments. We prove that the algorithm asymptotically converges to the optimal solution.

The paper is organized as follows. We start with a simple example to motivate this problem. Then in Section III we state the model and define notation. Based on the model, we derive the algorithm in Section IV. We analyze its optimality in Section V. We conclude the paper with Section VI.

\section{MOTIVATING EXAMPLE}

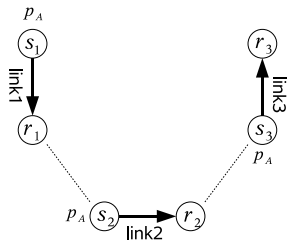

Fig. 1. Three Link Example (a): All three links use the same transmit power level $p_{A}$. Assume link 2 interferes with link 1 , and link 3 interferes with link 2 . Then only link 1 and link 3 can send packets concurrently at power level $p_{A}$.

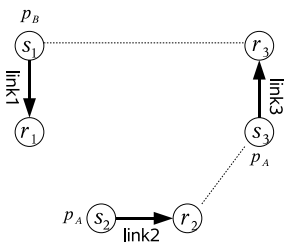

Fig. 2. Three Link Example (b): Link 1 increases its transmit power level to $p_{B}$, where $p_{B}>p_{A}$. Assume $p_{B}$ is selected such that link 2 does not interfere with link 1. However, link 1 could interfere with link 3 if $p_{B}$ is large.

To provide some intuition for how transmit power settings affect the throughput region, we start with a simple network. Consider the scenario in Figure 1. Without loss of generality, let the capacity of each link $l$ be 1 . Assume that when all three links select the same power to transmit packets, there are two interference-free transmission states: either link 1 and link 3 transmit concurrently, or link 2 transmits alone. Let vector $x_{1}=[1,0,1]$ and vector $x_{2}=[0,1,0]$ represent the above two states respectively, where the value 1 (or 0 ) indicates the corresponding link is active (or inactive). For instance, $x_{1}(1)=1$ means that link 1 is active in state 1 . Thus, the network can achieve a throughput region by timesharing between the two states. More formally, the maximum throughput under the power setting is characterized as the set of all convex combinations of the two vectors, i.e.,

$$
\vec{\lambda}_{\left[p_{A}, p_{A}, p_{A}\right]}=\alpha_{1}[1,0,1]+\alpha_{2}[0,1,0],
$$

where $\alpha_{1}+\alpha_{2}=1$, with $\alpha_{1}, \alpha_{2} \geq 0$. If the traffic arrival rate is within this region, then it is possible to serve it; otherwise, it is not possible to serve it. For example, if we ignore the scheduling overhead, the network in Figure 1 can serve traffic of rate $[0.5,0.5,0.5]$ but cannot serve $[1.0,0.5,0.5]$.

Now assume link 1 can transmit at a higher power level $p_{B}$. When it selects $p_{B}$, the transmission of link 2 cannot interfere with link 1's transmission because the received power at $r_{1}$ is larger. However, increasing transmit power for a node may cause interference to others. We assume that when link 1 uses transmit power $p_{B}$, it interferes with link 3 , as shown in Figure 2. One can see that now link 1 and link 2 can transmit concurrently without interfering with each other, but link 3 should not transmit when either link 1 or link 2 are transmitting, because of interference. Thus, the maximum throughput region consists of vectors of the form:

$$
\vec{\lambda}_{\left[p_{B}, p_{A}, p_{A}\right]}=\alpha_{1}[1,1,0]+\alpha_{2}[0,0,1] .
$$

One may ask which power level is better for link $1, p_{A}$ or $p_{B}$ ? Indeed, we cannot tell which one is better because both regions contain some points that the other does not contain. Therefore, any fixed power setting (either $\left[p_{A}, p_{A}, p_{A}\right]$ or $\left[p_{B}, p_{A}, p_{A}\right]$ ) is not throughput optimal.

Actually, the toy network can achieve the optimal throughput if the transmit power of link 1 is allowed to switch between $p_{A}$ and $p_{B}$. When the power selection scheme cooperates with the transmission scheduling perfectly, the maximum throughput consists of vectors of the form:

$$
\begin{aligned}
\vec{\lambda}_{\left[p_{A} \cup p_{B}, p_{A}, p_{A}\right]} & =\beta_{1} \vec{\lambda}_{\left[p_{A}, p_{A}, p_{A}\right]}+\beta_{2} \vec{\lambda}_{\left[p_{B}, p_{A}, p_{A}\right]} \\
& =\gamma_{1}[1,0,1]+\gamma_{2}[1,1,0],
\end{aligned}
$$

where $\beta_{1}+\beta_{2}=1$, with $\beta_{1}, \beta_{2} \geq 0$, and $\gamma_{1}+\gamma_{2}=1$, with $\gamma_{1}, \gamma_{2} \geq 0$. Obviously, this throughput contains both forms of vectors $\vec{\lambda}_{\left[p_{A}, p_{A}, p_{A}\right]}$ and $\vec{\lambda}_{\left[p_{B}, p_{A}, p_{A}\right]}$. Moreover, it also contains some points that are not contained in either of them, e.g., $[1.0,0.5,0.5]$. Thus, the vectors of the form $\vec{\lambda}_{\left[p_{A} \cup p_{B}, p_{A}, p_{A}\right]}$ constitute the optimal throughput region for this network. This motivates our goal of fully utilizing available power levels, and thereby achieving the optimal throughput region in arbitrary networks.

\section{SYSTEM MODEL}

In general, interference is affected by several factors such as channel status, noise, distance and transmit power. In this paper, we concern ourselves only with transmit power and assume all other factors are static. Thus, the change of interference relationships is solely due to the change of transmit powers. We assume that a number of discrete power levels are available at each wireless network interface card. This is true of current hardware. For example, the Cisco Aironet 350 series cards allow the transmit power level to be set to one of $1,5,20,30,50$, and $100 \mathrm{~mW}$. More formally, we assume there are $K$ available power levels, and let $P$ denote the set of these power levels. We model a wireless network as the set of wireless links denoted by $V$, and set $N=|V|$. If each link independently selects its transmit power, there are $K^{N}$ possibilities for power settings. Denote the $m$-th power setting by a $N \times 1$ vector $\vec{p}^{m}=\left[p^{m}(1), p^{m}(2), \ldots, p^{m}(N)\right]$, where $p^{m}(i) \in P$.

As mentioned in Section I, [7], [9] use a Markov random field model to analyze CSMA-based wireless networks. In order to leverage this model, we propose a generic conflict graph $G^{\prime}$ to capture the interference relationships in a multipower network. With each physical link we associate multiple virtual links, where each virtual link corresponds to a power level that the physical link can select. Since these virtual 
links correspond to the same actual link, they cannot transmit concurrently, and should therefore be considered as neighbors conflicting with each other in $G^{\prime}$. If we let a vertex denote a virtual link in $G^{\prime}$, then an actual link is represented by a clique whose size is equal to the number of its power levels. We identify the conflicting neighbors of these virtual links, and add an edge between two vertices if they conflict. In this way, we construct a single graph $G^{\prime}\left(V^{\prime}, E^{\prime}\right)$ that characterizes the conflict relationships in a network with multiple powers. Note that $V^{\prime}=V \times P$. Let $I S^{\prime}$ be the class of independent sets in $G^{\prime}$, and set $M:=\left|I S^{\prime}\right|$. Denote by $x^{m}$ the $m$-th Independent Set. We will refer to $x^{m}$ as a transmission state. Associate $x^{m}$ with a $N$ by $K, 0-1$ matrix $X^{m}=\left[x_{i k}^{m}\right]$, where $i$ is the index of a link and $k$ is the index of the power level, where $x_{i k}^{m}=1$ if the vertex of link $i$ with power $p_{k}$ belongs to the $m$-th Independent Set, and $x_{i k}^{m}=0$ otherwise.

Note that although we investigate pairwise interference, the proposed method also applies to the cumulative-interference model. Because our scheme is actually based on independent sets and not really by the conflict graph, it works as long as independent sets can be identified under the considered interference model (including the cumulative-interference model). The reason we define a conflict graph is only for ease of presentation.

\section{Random Power Selection for Maximal THROUGHPUT}

\section{A. Basis of Random Power Access}

The idea for our random power selection scheme comes from the CSMA type random access protocols such as IEEE 802.11. In a CSMA-based network, each wireless link can instantaneously become aware of the transmissions of its conflicting neighbors through carrier sensing. We make a similar but stronger assumption that each virtual link can independently carrier-sense the transmission of any of its conflicting neighbors. Like IEEE 802.11, our scheme sets up a back-off timer $t_{i k}$ for each virtual link. The timer is set randomly from an exponential distribution with mean $1 / R_{i k}$. For each virtual link, if none of its conflicting neighbors is active, then it waits (or backs-off) for $t_{i k}$, and then starts its transmission. During the back-off if some conflicting neighbor starts transmitting, then the virtual link pauses its back-off, and resumes it only when none of its conflicting neighbors is active. Notice that because the back-off timer is chosen from a continuous-time distribution, the probability of two links having the same timer is zero, and hence there is no collision. This idealized continuous-time model removes the effect of packet collisions and is focused on the interaction between wireless links. Reference [9] shows that this model actually matches with the NS-2 simulator pretty well in terms of the throughput. The reason is that in a large CSMAbased network, the mutual interference is the dominating factor that limits the network throughput. We also assume that the transmission time is exponentially distributed with mean 1. Based on these assumptions, we adopt the CSMA Markov chain model to solve our problem.

\section{B. Idealized Markov Chain Model}

This subsection introduces the idealized CSMA Markov chain model that has been recently developed in [7], [9]. We use this model to analyze our power selection scheme.

We model packet transmission time as an exponentially distributed random variable with mean 1 . Since the back-off timer $t_{i k}$ and transmission time are both continuous exponentially distributed random variables with means $1 / R_{i k}$ and 1 and independent, the transmission states form a continuous-time Markov chain. In state $x^{m}$, suppose $x_{i k}^{m}=0$ and none of its conflicting neighbors is active. Then $x_{i k}^{m}$ transitions to 1 (become active) at rate $R_{i k}$, which makes $x^{(m+1)}$ the same as $x^{m}$ at all components except that $x_{i k}^{(m+1)}=1$. Similarly, if $x_{i j}^{m}=1$ at state $x^{m}$, then $x^{m}$ will transition to $x^{(m-1)}$ at rate 1 , where $x^{(m-1)}$ is the same as $x^{m}$ at all components except $x_{i j}^{(m-1)}=0$.

Following the same argument as in [9], [7], the detailed balance property holds in this Markov Chain. Moreover, reference [9] points out that due to the Markov random field property the stationary distribution can be written as a product form, as follows. Denote $r_{i k}=\log R_{i k}$. Let $\vec{p}=\left[p_{1}, p_{2}, \ldots, p_{M}\right]$ be the stationary distribution of the Markov Chain. From detailed balance, for all $m \in I S^{\prime}$,

$$
p_{m}(\mathbf{r})=\frac{\exp \left(\sum_{i=1}^{N} \sum_{k=1}^{K} x_{i k}^{m} r_{i k}\right)}{B(\mathbf{r})},
$$

where

$$
B(\mathbf{r})=\sum_{n=1}^{M} \exp \left(\sum_{i=1}^{N} \sum_{k=1}^{K} x_{i k}^{n} r_{i k}\right) .
$$

From the distribution of transmission state, one can compute the normalized throughput (or service rate) of link $i$ at $p_{k}$ as

$$
s_{i k}=\sum_{m=1}^{N} x_{i k}^{m} p_{m}(\mathbf{r})
$$

and the total throughput of link $i$ as

$$
s_{i}=\sum_{k=1}^{K} s_{i k} .
$$

\section{Problem Formulation}

We study single-hop traffic, that is, packets independently arrive to each link, and once they are served, they immediately leave the system. We assume the traffic arrivals are Poisson processes which are independently across links. Let $\lambda_{i}$ denote the average arrival rate at link $i$, and $\vec{\lambda}=\left[\lambda_{1}, \lambda_{2}, \ldots, \lambda_{N}\right]$ denote the arrival rate vector of the network. We say that $\vec{\lambda}$ is feasible if and only if there exists $\left\{q_{m} \geq 0, \forall m\right\}$ with $\sum_{m=1}^{M} q_{m}=1$ such that

$$
\lambda_{i} \leq \sum_{k=1}^{K} \sum_{m=1}^{M} x_{i k}^{m} q_{m}, \forall i \in V .
$$

We call $\vec{q}$ the target transmission state distribution for $\vec{\lambda}$. The goal of our algorithm is to select $\mathbf{r}$ so that the actual distribution of the state converges to the target distribution. 


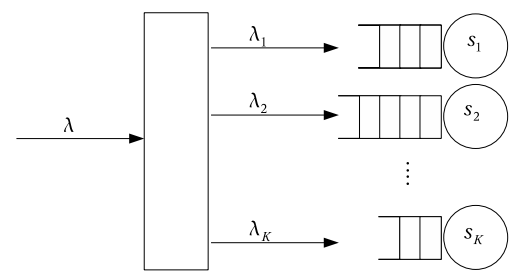

Fig. 3. System Model: The arrival rate is split into $K$ sub-rates, one for each power level. Also, each link maintains $K$ separate queues, one for each power level.

To this end, let us consider the following optimization problem:

$$
\begin{aligned}
\max & \sum_{m} \hat{q}_{m} \log p_{m}(\mathbf{r}) \\
\text { subject to } & \sum_{m} x_{i k}^{m} \hat{q}_{m} \geq \lambda_{i k}, \forall i, k \\
& \sum_{k}^{m} \lambda_{i k}=\lambda_{i}, \forall i \\
& \hat{q}_{m} \geq 0, \lambda_{i k} \geq 0, \forall i, k, m,
\end{aligned}
$$

where $\lambda_{i k}$ and $\hat{q}_{m}$ are decision variables. In (11), the $\lambda_{i}$ 's are link arrival rates, which are given. Note that it is not necessary that the summation of $\hat{q}_{m}$ be equal to 1 . We denote by $\vec{\lambda}$ the vector $\left[\lambda_{i}\right]$, and by $\hat{\lambda}$ the array $\left[\lambda_{i k}\right]$.

Theorem 1: If $\vec{\lambda}$ is feasible, i.e., satisfying constraint (8), then the optimal solution $\left(\mathbf{r}^{*}, \hat{\mathbf{q}}^{*}, \widehat{\lambda}^{*}\right)$ for the problem (9) (11) yields a service rate $s_{i}\left(\mathbf{r}^{*}\right)=\sum_{k} s_{i k}\left(\mathbf{r}^{*}\right)$ for each $i$ that is not less than the arrival rate $\lambda_{i}$.

We refer the readers to [4] for the proof of this theorem.

Since (9) is concave, and both (10) and (11) are linear, the above is a convex optimization problem. Our goal is to design a fully distributed and easy-to-implement algorithm. Although the Lagrangian method can decompose the problem to some extent, it requires each subproblem to exchange Lagrange multiplers with others, which could incur large communication overhead. Moreover, some variables are not available to the system. For example, $\hat{q}$ is not easy to obtain locally. We address this problem in the next subsection.

\section{Distributed Algorithm for Maximal Throughput}

If we further assume that the arrival rate $\lambda_{i}$ has been split into $K$ sub-rates $\lambda_{i k}$, one for each available power, as shown in Figure 3, with $\sum_{k} \lambda_{i k}=\lambda_{i}$, then the problem is identical to the one studied in [7]. However, the definition of feasible arrival rate vector has to be changed as follows. We say $\widehat{\lambda}:=$ $\left\{\lambda_{i k} \mid i=1,2, \ldots, N, k=1,2, \ldots, K\right\}$ is feasible if and only if there exists $\left\{q_{m} \geq 0, \forall m\right\}$ with $\sum_{m=1}^{M} q_{m}=1$ such that

$$
\lambda_{i k} \leq \sum_{m=1}^{M} x_{i k}^{m} q_{m}, \forall i \in V, \forall k \in P .
$$

This feasibility requirement is much stronger than (8). In other words, the arrival rate vector that is feasible for the network may not be feasible for (12) if the rate is not properly split. We need to first solve the simple problem in which the arrival traffic for each power level is given and feasible, and later consider the problem of how to split the arrival traffic.

For the system that has fixed arrival rate for each power level, the arrival traffic has to satisfy the constraint (12) so that the network can be stabilized. Stability requires that the service rate must be no less than the arrival rate. Comparing (6) with (12), one can see that the problem is essentially equivalent to making $\vec{p}$ equal to $\vec{q}$. This can be regarded as a KullbackLeibler distance minimization problem:

$$
\max _{\mathbf{r} \geq 0} F(\mathbf{r})=\sum_{m} q_{m} \log \left(p_{m}(\mathbf{r})\right) .
$$

Since both $\vec{q}$ and $\vec{p}$ are probability distributions, $F(\mathbf{r})$ is maximized when $\vec{q}=\vec{p}$. Also, $F(r)$ is concave in $\mathbf{r}$. Therefore, we can use a gradient method. Rewriting $F(\mathbf{r})$ gives

$$
\begin{aligned}
F(\mathbf{r}) & =\sum_{m} q_{m}\left(\sum_{i} \sum_{k} x_{i k}^{m} r_{i k}-\log B(\mathbf{r})\right) \\
& =\sum_{i} \sum_{k} \lambda_{i k} r_{i k}-\log \left(\sum_{n=1}^{M} \exp \left(\sum_{i=1}^{N} \sum_{k=1}^{K} x_{i k}^{n} r_{i k}\right)\right) .
\end{aligned}
$$

Differentiating it with respect to $r_{i k}$, we have

$$
\begin{aligned}
\frac{\partial F(\mathbf{r})}{\partial r_{i k}} & =\lambda_{i k}-\frac{\sum_{m} x_{i k}^{m} \exp \left(\sum_{i} \sum_{k} x_{i k}^{m} r_{i k}\right)}{B(\mathbf{r})} \\
& =\lambda_{i k}-s_{i k}(\mathbf{r}) .
\end{aligned}
$$

Therefore, a simple gradient algorithm to solve (13) is

$$
r_{i k}(t+1)=r_{i k}(t)+\alpha(t)\left(\lambda_{i k}-s_{i k}(t)\right),
$$

where $\alpha>0$. This is the algorithm used by Jiang and Walrand [7]. They also present a variant of the algorithm that sets $r$ proportional to the queue size, because there is similarity between queue evolution and $r$ 's update equation (16). Notice that this algorithm is simple and requires only local information. Thus, it is a distributed algorithm and easy to implement. Moreover, the algorithm is throughput optimal [7].

\section{E. Distributed Algorithm for Optimal Traffic Splitting}

Consider the optimization problem (9) - (11). Suppose we choose some $\hat{\lambda}$ satisfying (11), and set

$$
\hat{\mathbf{q}}:=\inf \left\{\mathbf{q} \mid \sum_{m} x_{i k} q_{m} \geq \lambda_{i k}, \forall i, k\right\} .
$$

If it turns out that $\sum_{n} \hat{q}_{n} \leq 1$, then the split according to $\widehat{\lambda}$ is a feasible solution and we are done. However, in general we may have $\sum_{n} \hat{q}_{n}>1$. If we now fix this $\hat{\mathbf{q}}$ and employ the gradient algorithm (16), then it will drive $p_{m}(\mathbf{r})$ to $\hat{q}_{m} / \sum_{n} \hat{q}_{n}$. Simultaneously, the service rate will be driven to

$$
s_{i k}=\frac{\sum_{m} x_{i k}^{m} \hat{q}_{m}}{\sum_{n} \hat{q}_{n}} .
$$

We want $\hat{q}_{m}$ to be as small as possible. However, it is lowerbounded by $\lambda_{i k}$. Thus, some constraints must be tight, i.e., for some $i, k, \sum_{m} x_{i k} \hat{q}_{m}=\lambda_{i k}$. Substituting these "tight" constraints into (17), we have $s_{i k}=\lambda_{i k} / \sum_{n} \hat{q}_{n}<\lambda_{i k}$. This 
observation suggests that $\left(s_{i k}-\lambda_{i k}\right)$ is a good indicator of the tightness of the resource. In particular, if $s_{i k}-\lambda_{i k}<0$, it implies that this constraint is tight, and we need to shift some traffic away from $\lambda_{i k}$; on the other hand, if $s_{i k}-\lambda_{i k}>0$, it means that this constraint is "loose," and we can shift some traffic into it.

Based on this observation, we have the following traffic splitting algorithm:

$$
\lambda_{i k}(t+1)=\lambda_{i k}(t)+c_{i k}(t)\left[s_{i k}(t)-\lambda_{i k}(t)\right], \forall i, k,
$$

where $c_{i k}(t)$ is some small positive step size.

Note that each time we update $\lambda_{i k}$, the constraint (11) has to hold. Thus, $c_{i k}(t)$ should be chosen such that

$$
\sum_{k} c_{i k}(t)\left[s_{i k}(t)-\lambda_{i k}(t)\right]=0, \forall i .
$$

In summary, our random power access scheme is a combination of two algorithms, one adapts $\mathbf{r}$, and the other splits $\vec{\lambda}$. In particular, each virtual link adaptively updates $\mathbf{r}(t)$ by the algorithm given by (16). For an actual link, if some $s_{i k}(t)-\lambda_{i k}(t)$ is positive while others are negative, then the algorithm given by (18) is invoked to shift traffic among virtual links of this actual link. In the next section, we show that, when combined, the two algorithms converge to the optimal solution, and hence achieve the ultimate capacity region.

\section{AnAlysis of the Algorithms}

We have present a distributed power control scheme that consists of two algorithms operating in parallel. Between them, we obtain the traffic shifting algorithm (18). The algorithms have been designed individually, and what we now analyze is how they interact with each other when we jointly use both of them. In this section we show that they converge to the optimal point.

We first present LaSalle's invariance principle that is useful in analyzing the asymptotic behavior of differential equations [8]. It states that for the differential equation $\dot{\mathbf{x}}(t)=f(\mathbf{x}(t))$, if there exists a radially unbounded, continuously differentiable, positive definite function $Y: \mathcal{D} \rightarrow \mathcal{R}$ such that $\dot{Y}(\mathbf{y}) \leq 0$ for all $\mathbf{y} \in \mathcal{D}$, then every solution starting in $\mathcal{D}$ approaches the set $\mathcal{M}$ as $t \rightarrow \infty$, where $\mathcal{M}$ is the largest invariant set contained in the points where $\dot{Y}(\mathbf{y})=0$.

To utilize this theorem, we assume that the updating speed is such that the evolutions of $\mathbf{r}$ and $\hat{\lambda}$ are characterized by the following continuous-time differential equations

$$
\dot{r}_{i k}(t)=\alpha(t)\left(\lambda_{i k}(t)-s_{i k}(t)\right)
$$

and

$$
\dot{\lambda}_{i k}(t)=c_{i k}(t)\left(s_{i k}(t)-\lambda_{i k}(t)\right),
$$

for each $i, k$. Then, the following global asymptotic stability result holds.

Theorem 2: If $\vec{\lambda}$ is feasible, i.e., it satisfies constraint (8), then by algorithms (16) and (18), the system $(\mathbf{r}(t), \widehat{\lambda}(t))$ converges to an invariant set consisting of $\left(\mathbf{r}^{*}, \widehat{\lambda}^{*}\right)$ such that $\sum_{k} s_{i k}\left(\mathbf{r}^{*}\right)=\lambda_{i}$, for all $i \in V$.

We refer the readers to [4] for further details.

\section{CONCLUSION}

We have presented a distributed randomized power control scheme that achieves the ultimate optimal throughput capacity in a wireless network where multiple power levels are available to each node. The randomized scheme outperforms any fixed power selection scheme since it generally has a larger feasible throughput region. We prove that the nonlinearly coupled system asymptotically converges to the global optimum of the joint power control and multiple access control. Through the paper, we expand the scope of the random access control method to encompass the power control problem. This enables us to combine MAC layer with the PHY layer to achieve a larger region of network capacity.

\section{REFERENCES}

[1] M. Burkhart, P. Rickenbach, R. Wattenhofer, and A. Zollinger. "Does topology control reduce interference?". In ACM MobiHoc, 2004.

[2] A. Eryilmaz, A. Ozdaglar, and E. Modiano. "Polynomial complexity algorithms for full utilization of multi-hop wireless networks". In IEEE Infocom, 2007.

[3] Y. Gao, J. C. Hou, and H. Nguyen. "Topology control for maintaining network connectivity and maximizing network capacity uder the physical model". In IEEE Infocom, 2008.

[4] Y. Gao, Z. Zeng, and P. R. Kumar. "joint random access and power selection for maximal throughput in wireless networks". Technical report, Coordinated Science Lab., University of Illinois, Urbana-Champaign, December 2009

[5] P. Gupta and P. R. Kumar. "The capacity of wireless networks". IEEE Trans. Infom. Theory, 46:388 - 404, 2000.

[6] K. Jain, J. Padhye, V. N. Padmanabhan, and L. Qiu. "Impact of interference on multi-hop wireless network performance". In $A C M$ Mobicom, 2003.

[7] L. Jiang and J. Walrand. "A distributed csma algorithm for throughput and utility maximization in wireless networks". In Allerton Conference on Communication, Control, and Computing, 2008.

[8] H. Khalil. Nonlinear System. Upper Saddle River, 3rd edition, 2002.

[9] S. C. Liew, C. Kai, J. Leung, and B. Wong. "Back-of-the-envelope computation of throughput distribution in csma wireless networks". submitted for publication, http://arxiv.org/abs/0712.1854.

[10] N. McKeown, A. Mekkittikul, V. Anantharam, and J. Walrand. "Achieving 100 throughput in an input-queued switch". IEEE Trans. on Communications, 1999.

[11] E. Modiano, D. Shah, and G. Zussman. "Maximizing throughput in wireless networks via gossiping”. In ACM SIGMETRICS, 2006.

[12] T. Moscibroda, R. Wattenhofer, and A. Zollinger. "Topology control meets sinr: the scheduling complexity of arbitrary topologies". In $A C M$ MobiHoc, 2006.

[13] S. Sanghavi, L. Bui, and R. Srikant. "Distributed link scheduling with constant overhead". In ACM SIGMETRICS, 2007.

[14] L. Tassiulas. "Linear complexity algorithms for maximum throughput in radio networks and input queued switch". In IEEE Infocom, 1998.

[15] L. Tassiulas and A. Ephremides. "Stability properties of constrained queueing system and scheduling policies for maximum throughput in multihop radio networks". IEEE Trans. on Automatic Control, 36:19361948, 1992. 